\title{
Determinação do Perfil Ótimo de Alimentação de Substrato no Processo de Fermentação Alcoólica - Influência da Condição Inicial
}

F.S. LOBATO ${ }^{1}$, Faculdade de Engenharia Química, Universidade Federal de Uberlândia, Campus Santa Mônica, 38400-902 Uberlândia, MG, Brasil.

\begin{abstract}
Resumo. A otimização dinâmica do processo de fermentação batelada alimentada, através do controle da vazão de alimentação de substrato, caracteriza um problema de controle singular de difícil solução devido à flutuação do índice diferencial e da ativação das restrições. Nesse caso, a estratégia ótima de alimentação é obtida através da aplicação do Princípio de Pontryagin e de procedimentos de redução de índices superiores para a determinação da Função Identificadora de Fases. Dessa forma, o problema original é transformado em um problema de identificação dos instantes em que as fases ocorrem e da estratégia de controle em cada fase. No presente trabalho é avaliado o efeito da concentração inicial de substrato dentro do fermentador sobre os perfis ótimos de alimentação. Os resultados mostram diferentes estratégias de alimentação de substrato em função da concentração inicial de substrato no meio fermentativo, configurando assim novas possibilidades para a otimização do processo em escala industrial.
\end{abstract}

Palavras-chave. Problema de controle ótimo, arco singular, fermentação alcoólica.

\section{Introdução}

O Problema de Otimização Dinâmica ou Problema de Controle Ótimo (PCO) consiste na obtenção dos perfis das variáveis de controle que maximizam ou minimizam um determinado índice de desempenho $[3,5,8]$. No caso específico do processo de fermentação batelada alimentada, deseja-se maximizar a produtividade de etanol através da manipulação da taxa de alimentação de substrato. Esse PCO é caracterizado pela presença de singularidades devido à flutuação do índice diferencial e de ativações de restrições na variável de controle e no volume do fermentador.

Para resolver este problema, vários métodos numéricos têm sido propostos [12, 10, 5, 8]. Estes são classificados em $[3,7,10,5,8]$ : métodos diretos, métodos baseados na aplicação do Princípio de Pontryagin, conhecido popularmente como métodos indiretos e o método HJB (Hamilton-Jacobi-Bellman).

Tradicionalmente, os métodos diretos têm sido usado para a resolução do PCO devido a sua facilidade de implementação e por não demandar a geração das condições de otimalidade e posterior resolução de um Problema de Valor no Contorno

\footnotetext{
${ }^{1}$ fslobato@feq.ufu.br. O autor agradece o apoio da FAPEMIG pelo suporte financeiro deste trabalho.
}

Recebido em 26 Agosto 2010; Aceito em 13 Abril 2011. 
Algébrico-Diferencial (PVCAD) [12]. Já os métodos indiretos, consistem na transformação do problema de otimização original em um PVCAD resultante da aplicação das condições de otimalidade. Como principais dificuldades pode-se citar a dificuldade da geração das condições de otimalidade para sistemas de grande dimensão e da dificuldade inerente da resolução do problema de valor no contorno resultante $[3,4,7,10]$. Apesar destes obstáculos, a abordagem indireta tem sido aplicada nos processos de fermentação $[13,14,9]$ para a obtenção da Função Identificadora de Fases (FIF) [10] necessária à determinação da estratégia ótima de alimentação de substrato.

O problema de fermentação batelada alimentada é caracterizado por três fases básicas: modo de operação batelada (sem entrada e saída de massa através do volume de controle considerado) e dois modos de operação batelada alimentada (o controle operando na condição de saturação, isto é, vazão máxima ou o controle singular) $[13,14,9,11,5,8]$. A escolha do modo de operação inicial caracterizase como uma das principais dificuldades. Nos últimos anos, a análise física do problema, aliado a escolha das condições iniciais e da avaliação da FIF, tem se configurado como um bom indicativo do modo de operação a ser implementado no início do processo $[13,14]$.

Neste sentido, este trabalho apresenta uma sistematica geral para a solução de PCO singulares cujos perfis de controle estão sujeitos a restrições de trajetória. São realizadas avaliações do efeito da concentração inicial de substrato dentro do fermentador sobre a FIF e da estratégia de controle obtida.

\section{O Processo de Fermentação Batelada Alimen- tada}

A fermentação é um dos processos químicos mais antigos já desenvolvidos pelo homem. No meio industrial, é um dos principais processos, cuja aplicabilidade pode ser comprovada em diversas áreas, tais como na produção de bebidas alcoólicas, vinagres, etanol, ácidos orgânicos, biopolímeros, solventes, vitaminas, antibióticos, entre outras aplicações [2].

Conceitualmente, o processo de fermentação consiste de uma complexa transformação de nutrientes de um meio de cultura, pela ação metabólica dos microrganismos presentes, em produtos e mais células microbianas como descrito a seguir:

$$
\text { células }+ \text { nutrientes } \rightarrow \text { produtos }+ \text { mais células }+ \text { energia }
$$

No contexto de otimização, deseja-se maximizar a quantidade de etanol produzido a partir de glicose via ação de microorganismos e sujeito às equações de balanço de massa

$$
\dot{\mathbf{x}}=f \rightarrow\left[\begin{array}{c}
\dot{x} \\
\dot{s} \\
\dot{p} \\
\dot{v}
\end{array}\right]=\left[\begin{array}{c}
\mu x-\frac{u}{v} x \\
-\sigma x+\frac{u}{v}\left(s_{f}-s\right) \\
\pi x-\frac{u}{v} p \\
u
\end{array}\right], \quad \mathbf{x}(0)=\left[\begin{array}{c}
x_{o} \\
s_{o} \\
p_{o} \\
v_{o}
\end{array}\right]
$$


onde $x, s$ e $p$ são as concentrações de células, substrato e produto, em unidades de massa por unidade de volume, respectivamente, $u$ é a taxa de alimentação de substrato em unidades de volume por unidade de tempo, $s_{f}$ é a concentração da corrente de substrato alimentado em unidades de massa por unidade de volume, $v$ é o volume do fermentador, $\mu$ e $\sigma$ são as expressões da taxa de crescimento específica para células e substrato, ambas por unidade de tempo, e $\pi$ é produtividade específica por unidade de tempo.

Fisicamente são impostas restrições na variável de controle $(u)$, no volume $(v)$ e no rendimento teórico $(\eta)$, respectivamente

$$
\begin{gathered}
0=u_{\min } \leq u \leq u_{\max }, \\
v \leq v_{\max }, \\
\frac{p v-p_{o} v_{o}}{\left(v-v_{o}\right) s_{f}+v_{o} s_{o}-v s} \leq \eta .
\end{gathered}
$$

\section{Formulação Matemática do Problema de Con- trole Ótimo}

A formulação matemática de um PCO genérico é dado por [3]:

$$
\begin{gathered}
\min J=\psi\left(\mathbf{x}\left(t_{f}\right), t_{f}\right)+\int_{t_{0}}^{t_{f}} L(\mathbf{x}, \mathbf{u}, t) d t \\
f(\dot{\mathbf{x}}, \mathbf{x}, \mathbf{u}, t)=0 \\
S(\mathbf{x}, \mathbf{u}, t) \leq 0 \\
\varphi\left(\mathbf{x}\left(t_{f}\right)\right)=0 \\
\mathbf{x}^{L} \leq \mathbf{x} \leq \mathbf{x}^{U} \\
\mathbf{u}^{L} \leq \mathbf{u} \leq \mathbf{u}^{U}
\end{gathered}
$$

onde $\mathbf{x}$ é o vetor das variáveis de estado, $\mathbf{u}$ é o vetor das variáveis de controle, $\psi\left(\mathbf{x}\left(t_{f}\right), t_{f}\right)$ é o primeiro termo da função objetivo avaliado em $t=t_{f}$ e $L(\mathbf{x}, \mathbf{u}, t)$ é o segundo termo do funcional $J, S(\mathbf{x}, \mathbf{u}, t)$ é o vetor de restrições de desigualdade, $f(\dot{\mathbf{x}}, \mathbf{x}, \mathbf{u}, t)$ é o vetor de restrições algébrico-diferenciais, e $\varphi\left(\mathbf{x}\left(t_{f}\right)\right)$ é o vetor de variáveis definidas no tempo final $t_{f}$. Os sobrescritos $L$ e $U$ identificam, respectivamente, os limites inferior e superior das variáveis.

\subsection{Solução do PCO através da abordagem indireta}

Os métodos indiretos surgiram com o desenvolvimento do Cálculo Variacional, permitindo a dedução das condições necessárias e suficientes para a solução de PCOs $[3,8]$. Tal metodologia consiste da transformação do problema original em um PVCAD e posterior resolução por uma dada técnica numérica [6]. 
Seja o problema de controle descrito pelas equações (3.1) e (3.2). Para este sistema, a função Hamiltoniano é definida como [3, 7, 10]:

$$
H \equiv L(\mathbf{x}, \mathbf{u}, t)+\lambda^{T} f .
$$

A partir da aplicação do Princípio de Pontriagyn, obtemos as condições de otimalidade ou Equações de Euler-Lagrange [3, 7, 10]:

$$
\begin{gathered}
\dot{\lambda}=-\frac{\partial H}{\partial \dot{\mathbf{x}}}, \quad \lambda\left(t_{f}\right)=\frac{\partial \psi\left(\mathbf{x}\left(t_{f}\right), t_{f}\right)}{\mathbf{x}\left(t_{f}\right)}, \\
\frac{\partial H}{\partial \mathbf{u}}=0,
\end{gathered}
$$

onde a equação (3.9) é conhecida como condição estacionária.

\subsection{O PCO linear na variável de controle}

A Teoria de Controle Ótimo diz que a estratégia de controle ótimo para o PCO linear com relação à variável de controle deve seguir a seguinte relação matemática [3]:

$$
u(t)= \begin{cases}u_{\max } & \text { se } \mathrm{FIF}<0 \\ u_{\text {sing }} & \text { se } \mathrm{FIF}=0 \\ u_{\min } & \text { se } \mathrm{FIF}>0\end{cases}
$$

onde FIF $\equiv \frac{d^{n}}{d t^{n}}\left(\frac{\partial H}{\partial \mathbf{u}}\right)$ e $n$ é o número de diferenciações necessárias para explicitar a variável de controle.

Desta maneira faz-se necessário o conhecimento da Função Identificadora de Fases (FIF) [10] para a determinação de qual estratégia de controle será empregada na fase corrente.

Destacada uma das principais dificuldade referentes à resolução do PCO via abordagem indireta, será apresentada na seção 4., a metodologia para a obtenção da FIF em função apenas das variáveis de estado, evitando assim a resolução do problema de valor no contorno oriundo da aplicação do Princípio de Pontriagyn.

\subsection{Arco singular e índice diferencial}

Conceitualmente, arcos singulares ocorrem quando a matriz Hessiana do Hamiltoniano com relação às variáveis de controle é singular [3]. No caso específico do problema de fermentação, a presença da variável de controle na forma linear introduz naturalmente singularidade a esse problema, assim como destacado na seção anterior.

Já os PCOs não lineares podem apresentar impulsos nas variáveis de controle se elas não forem submetidas a limites. Quando estes limites são impostos, a solução apresentará períodos com a variável de controle nos limites de restrição e arcos 
singulares [3]. Frequentemente as variáveis de controle apresentam descontinuidades quando passam do arco restrito para o arco singular ou vice-versa $[3,10,11]$.

Outra definição relevante na análise de PCOs é o conceito de Índice Diferencial (ID). Segundo Brenan et al [4], o ID é o número mínimo de vezes que o sistema algébrico-diferencial ou parte dele deve ser diferenciado com relação ao tempo para determinação de um sistema puramente diferencial. Tal conceito está diretamente relacionado ao grau de dificuldade de resolução de um sistema algébrico-diferencial, decorrente de mau condicionamento, instabilidade, singularidade e má convergência. O ID é uma quantidade local, isto é, definida num ponto particular da trajetória de estados e pode mudar em pontos discretos ao longo desta trajetória.

\section{Metodologia}

Assim como mencionado anteriormente, esta seção é dedicada ao desenvolvimento de uma metodologia para a obtenção da FIF em função apenas das variáveis de estado. Neste contexto, redefinindo como variável de controle a taxa de diluição $(D \equiv u / v)$, ao invés da vazão de alimentação de substrato, consegue-se eliminar estrategicamente a equação do balanço global $(\dot{v}=u)$ do sistema dado por $(2.1)$ :

$$
\left[\begin{array}{c}
\dot{x} \\
\dot{s} \\
\dot{p}
\end{array}\right]=\left[\begin{array}{c}
\mu x \\
-\sigma x \\
\pi x
\end{array}\right]+\left[\begin{array}{c}
-x \\
\left(s_{f}-s\right) \\
-p
\end{array}\right] D \Rightarrow F=F_{0}+F_{1} D .
$$

Neste caso, a função Hamiltoniano $(H)$ é definida como sendo

$$
H \equiv \lambda^{T} F=\lambda^{T} F_{0}+\lambda^{T} F_{1} D .
$$

A condição estacionária (equação (3.9)) é dada por

$$
\lambda^{T} F_{1}=0 .
$$

Para problemas com tempo final livre sabemos que a função $H$ é nula na trajetória ótima de controle [3], mas como $\lambda^{T} F_{1}$ (condição estacionária) tem de ser nula, isso implica que $\lambda^{T} F_{0}$ também será nula,

$$
\lambda^{T} F_{0}=0 .
$$

Como as equações (4.3) e (4.4) são lineares com relação ao vetor de variáveis adjuntas $\lambda$, é possível escrever $\lambda_{1}=A_{1} \lambda_{3}$ e $\lambda_{2}=A_{2} \lambda_{3}$, onde $A_{1}$ e $A_{2}$ são funções apenas de variáveis de estado. A partir da diferenciação da condição estacionária, com relação ao tempo, e com a substituição de $\lambda_{1}\left(\mathbf{x}, \lambda_{3}\right)$ e de $\lambda_{2}\left(\mathbf{x}, \lambda_{3}\right)$ não é possível obtermos a variável de controle $D$ explicitamente na equação (4.5)

$$
\dot{\lambda}^{T} F_{1}+\lambda^{T} \dot{F}_{1}=\lambda_{3} A_{3}=0,
$$

onde $A_{3}$ é uma função apenas de variáveis de estado.

Como $\lambda_{3}$ não necessariamente é nula, implica que

$$
A_{3}=0 .
$$


A equação (4.6) é a FIF e que será utilizada para a identificação dos instantes de transição entre as fases (eventos) e a estratégia de controle em cada uma delas a partir da diferenciação da equação 4.6 com relação ao tempo

$$
D_{\text {sing }} \rightarrow \frac{d A_{3}}{d t}=\chi\left(\dot{\mathbf{x}}, \mathbf{x}, D_{\text {sing }}\right)=0 \text {. }
$$

Deve ser destacado que com essa manipulação algébrica, são necessárias 2 diferenciações para a obtenção explícita da variável de controle $D$. Para a obtenção do sistema puramente diferencial faz-se necessário a diferenciação da equação (4.7) com relação ao tempo, caracterizando assim um problema com índice diferencial igual a 3. Lembrando que quando a variável de controle $D$ for obtida, a equação de balanço global $(\dot{v}=u)$ e a taxa de alimentação de substrato $u$ são novamente inseridas no sistema original.

\section{Resultados e Discussões}

Neste trabalho são utilizadas as expressões para a taxa de crescimento específica $(\mu$ e $\sigma)$ e para a produtividade específica $\pi$ para o microorganismo Shaccaromyces cerevisae propostas por Aiba et al [1] $(0 \leq s \leq 120 \mathrm{~g} / \mathrm{L}$ e $0 \leq p \leq 50 \mathrm{~g} / \mathrm{L})$

$$
\mu=\frac{0,408 s \exp (-0,028 p)}{0,22+s}, \quad \pi=\frac{s \exp (-0,015 p)}{0,44+s}, \quad \sigma=10 \mu .
$$

Para avaliar a influência da concentração inicial de substrato $\left(s_{o}\right)$ no valor da FIF (equação (4.6)) e na estratégia de controle utilizada na fase atual, serão analisados os valores de $s_{o}$ que levam o processo a ser inicializado com $u=u_{\min }, u=u_{\max }$ ou $u=u_{\text {sing }}$. As outras condições iniciais e os parâmetros utilizados são: $x_{o}=0,2 \mathrm{~g} / \mathrm{L}$, $p_{o}=0 \mathrm{~g} / \mathrm{L}, v_{o}=5 \mathrm{~L}, u_{\max }=3 \mathrm{~L} / \mathrm{h}, v_{\max }=20 \mathrm{~L}, s_{f}=100 \mathrm{~g} / \mathrm{L}$ e $\eta=0,51$.

Substituindo as condições e os parâmetros na FIF (equação (4.6)), esta se torna uma função apenas de $s$. Assim podemos escolher $s_{o}$ de tal forma a termos $\mathrm{FIF}=0$, FIF $>0$ ou FIF $<0$. Desta maneira são consideradas três possibilidades para a inicialização do modo de operação:

- Caso A: $s_{o}=0 \mathrm{~g} / \mathrm{L}$, o que nos leva a inicializar o processo com $u=u_{\max }$. Fisicamente isto significa que devemos fazer com que a concentração de substrato aumente rapidamente para que as células possam crescer.

- Caso B: $s_{o}=100 \mathrm{~g} / \mathrm{L}$, neste caso, esse valor de $s_{o}$ faz com que a FIF seja nula. Assim, podemos inicializar o processo com $u=u_{\text {min }}$ (Caso B) ou $u=u_{\text {sing }}$ (Caso C). No Caso B, o processo é inicializado com modo de operação batelada já que $s_{o}$ é alto, fazendo com que as células cresçam rapidamente. Já no caso C, o controle é singular e dado pela equação (4.7). Em cada um dos casos, o processo é finalizado quando a concentração de substrato é zero $\left(s \approx 10^{-8}\right)$, definindo assim o tempo total de fermentação $\left(t_{f}\right)$.

Desse modo, de posse da FIF e da estratégia de controle com que o processo será inicializado, o problema original de otimização é convertido em um problema 
equivalente de identificação dos instantes em que ocorre a mudança de fase (eventos). O sistema formado pelas equações (2.1) a (2.4), a FIF (equação (4.6)) e o controle singular (equação (4.7)) são resolvidos pelo método Runge-Kutta $4^{a}$ ordem.

A Tabela 1 apresenta as estratégias de controle e respectivos índices diferenciais obtidos em cada uma das fases.

Tabela 1: Estratégia de controle $(u)$ e índice diferencial (ID) definido por fase.

\begin{tabular}{|c|c|c|c|c|c|}
\hline \multirow{2}{*}{ A } & $0<t \leq t_{s 1}$ & $t_{s 1}<t \leq t_{s 2}$ & $t_{s 2}<t \leq t_{s 3}$ & $t_{s 3}<t \leq t_{s 4}$ & $t_{s 4}<t \leq t_{f}$ \\
\hline & $u_{\max } \mathrm{e} \mathrm{ID}=1$ & $u_{\min } \mathrm{e} \mathrm{ID}=1$ & $u_{\operatorname{sing}}$ e ID $=3$ & $u_{\max } \mathrm{e} I \mathrm{ID}=1$ & $u_{\min }$ e ID $=1$ \\
\hline \multirow{2}{*}{ B } & $0<t \leq t_{s 1}$ & $t_{s 1}<t \leq t_{s 2}$ & $t_{s 2}<t \leq t_{s 3}$ & $t_{s 3}<t \leq t_{f}$ & \\
\hline & $u_{\min }$ e ID $=1$ & $u_{\text {sing }}$ e ID $=3$ & $u_{\max }$ e ID $=1$ & $u_{\min }$ e ID $=1$ & \\
\hline \multirow{2}{*}{$\mathrm{C}$} & $0<t \leq t_{s 1}$ & $t_{s 1}<t \leq t_{f}$ & & & \\
\hline & $u_{\text {sing }}^{*}$ e ID $=3$ & $u_{\min } \mathrm{e} \mathrm{ID}=1$ & & & \\
\hline
\end{tabular}

${ }^{*}$ Neste caso como $u_{\text {sing }} \geq u_{\max } \Rightarrow u_{\text {sing }}=u_{\max }$

Nessa tabela é possível observarmos que no caso A $\left(s_{o}=0 \mathrm{~g} / \mathrm{L}\right)$ o processo inicializa-se com $u=u_{\max }$ até que a FIF seja nula $\left(u=u_{\text {sing }}\right)$, mas como o controle calculado é menor que zero, nessa fase usa-se $u=u_{\min }$ até que a FIF se anule. A partir daí $u=u_{\text {sing }}$ até que que a restrição na variável de controle fique ativa $\left(u=u_{\max }\right)$. Esta fase é mantida até que a restrição no volume do reator seja satisfeita $\left(v=v_{\max }\right)$, com isso $u=u_{\min }$. No caso B $\left(s_{o}=100 \mathrm{~g} / \mathrm{L}\right) \mathrm{o}$ processo inicializa-se com $u={ }_{\min }$ até que a FIF seja nula $\left(u=u_{\text {sing }}\right)$. Essa fase finaliza-se quando a restrição no controle fica ativa, usando a partir desse instante $u=u_{\text {min }}$. No caso $\mathrm{C}\left(s_{o}=100 \mathrm{~g} / \mathrm{L}\right)$ o processo inicializa-se com $u=u_{\text {sing }}$, mas como $u_{\text {sing }}>u_{\max }$, implica que $u_{\text {sing }}=u_{\max }$ até que a restrição no volume seja alcançada $\left(v=v_{\max }\right)$, fazendo com que $u=u_{\min }$.

A Tabela 2 mostra os eventos, o tempo final e a produtividade obtida ao final do processo de fermentação para cada um dos casos. Nesta tabela, pode-se observar

Tabela 2: Eventos $\left(t_{s i}(\mathrm{~h})\right)$, tempo total de fermentação $\left(t_{f}(\mathrm{~h})\right)$ e produtividade $(p v(\mathrm{~g}))$ para os casos estudados.

\begin{tabular}{|c|c|c|c|c|c|c|}
\hline Caso & $t_{s 1}(\mathrm{~h})$ & $t_{s 2}(\mathrm{~h})$ & $t_{s 3}(\mathrm{~h})$ & $t_{s 4}(\mathrm{~h})$ & $t_{f}(\mathrm{~h})$ & $p v(\mathrm{~g})$ \\
\hline \hline A & 2,01 & 10,36 & 13,62 & 14,25 & 15,02 & 425,28 \\
\hline B & 11,41 & 18,66 & 19,34 & - & 19,99 & 670,19 \\
\hline C & 4,99 & - & - & - & 15,50 & 581,68 \\
\hline
\end{tabular}

que o caso B foi o que apresentou maior produtividade às custas de um maior tempo de fermentação. Comparando apenas A e C, os tempos de fermentação são próximos, mas a produtividade de $\mathrm{C}$ é superior a de $\mathrm{A}$.

As figuras 1 a 3 apresentam os perfis ótimos para a concentração de células $(x)$, 
de substrato $(s)$, de produto $(p)$, o volume $(v)$, a taxa de alimentação de substrato ( $u$ - estratégia de controle) e a produtividade $(p v)$ para cada um dos casos.

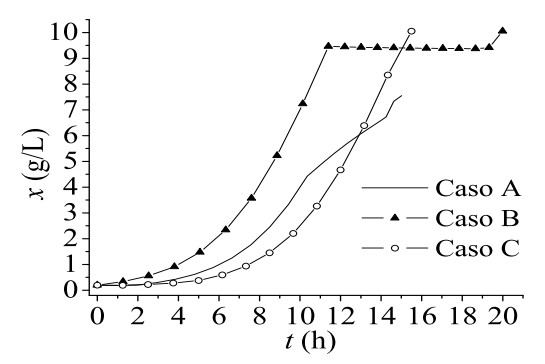

(a) $x(\mathrm{~g} / \mathrm{L})$.

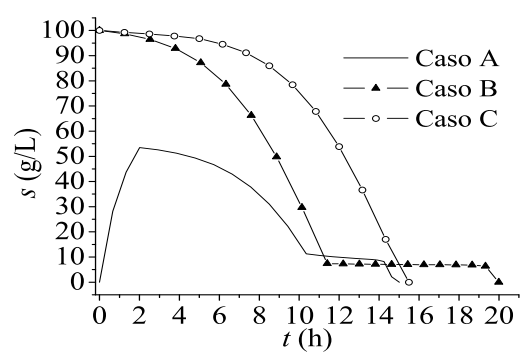

(b) $s(\mathrm{~g} / \mathrm{L})$.

Figura 1: Perfis de concentração de células e de substrato.

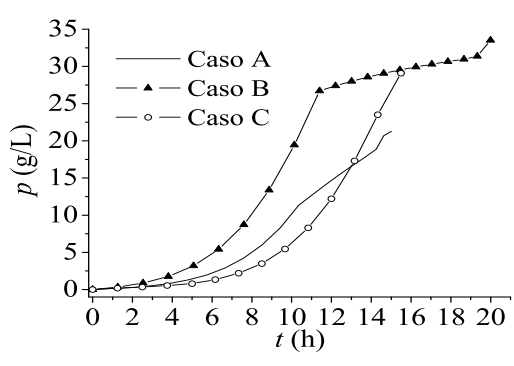

(a) $p(\mathrm{~g} / \mathrm{L})$.

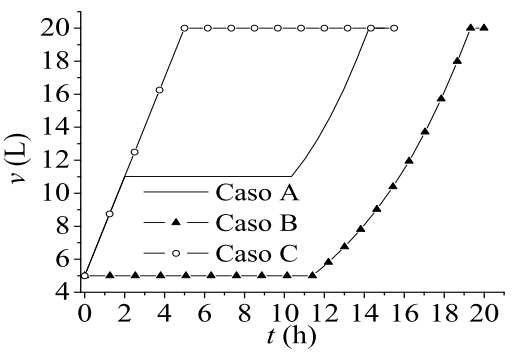

(b) $v(\mathrm{~L})$.

Figura 2: Perfis de concentração de produto e volume do fermentador.

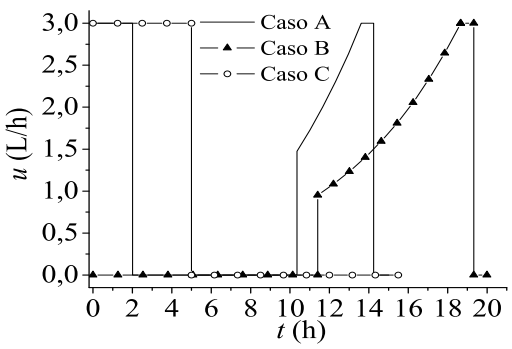

(a) $u(\mathrm{~L} / \mathrm{h})$.

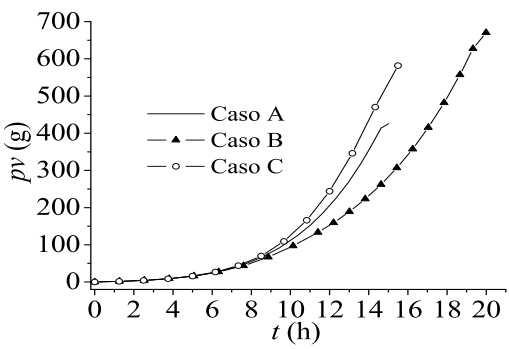

(b) $p v(\mathrm{~g})$.

Figura 3: Perfis da variável de controle e de produtividade.

Considerando a densidade da solução como sendo $1 \mathrm{~g} / \mathrm{L}$, a massa total de subs- 
trato alimentado nos casos A, B e C, é 228,33 g; 287,14 g e 231,88 g, respectivamente. Para as expressões de taxa de crescimento específica e de produtividade específica consideradas, percebe-se que quanto maior a massa de substrato alimentado, maior é a produtividade.

\title{
6. Conclusões
}

Este trabalho apresentou uma estratégia de redução da fase singular do PCO aplicado ao processo de fermentação batelada alimentada para produção de etanol, considerando restrições na produtividade, no controle, no volume do reator e tempo total de fermentação livre. A metodologia de solução empregada, em que modelos de valor inicial são resolvidos a cada fase, cujas características são definidas pelos respectivos índices e que necessitam da determinação dos vários eventos, apresentou resultados fisicamente satisfatórios. Todavia, devido à escolha oportuna dos parâmetros utilizados neste estudo, não foi possível comparar quantitativamente os resultados obtidos com aqueles reportados por Modak e Lim [14].

De forma geral, comparando as estratégias utilizadas no caso A com as utilizadas nos casos B e C, cada um dos perfis obtidos são soluções ótimas para a condição inicial considerada. Entretanto, quando confrontamos apenas os casos B e C $\left(s_{o}=100 \mathrm{~g} / \mathrm{L}\right)$ e que começam por $u=u_{\text {min }}$ e $u=u_{\text {sing }}$, respectivamente, a utilização de $u=u_{\text {min }}$ nos leva a um melhor resultado em termos de produtividade, mas com um maior tempo de fermentação (Tab. 1).

Como linha de trabalhos futuros pode-se citar a formulação de um PCO com condições iniciais a serem determinadas pela otimização, eliminando desta maneira tal dependência.

\begin{abstract}
The dynamic optimization of fermentation processes, through the control of the substrate feed rate, characterizes a singular control problem of difficult solution due to differential index fluctuation and constraints activation. In this case, the optimal feed strategy is obtained through the application of the Pontryagin' Principle and of the procedures for reduction of superior indexes through the Phases Identification Function. In this way, the original problem is transformed in a similar problem for events identification and for control strategy. The effect of substrate initial concentration in optimal profiles is evaluated. The results show that it is possible to obtain different strategies of substrate feed rate, configuring new alternatives for the use in industrial context.
\end{abstract}

Key-words: Optimal control problem, singular arc, alcohol fermentation.

\section{Referências}

[1] S. Aiba, M. Shoda, M. Nagatani, Kinetics of product inhibition in alcohol fermentation, Biotechnology and Bioengineering, X (1968), 845-864 (reprinted in Biotechnology and Bioengineering, 67, 2000).

[2] P.C.S. Borges, "Otimização Dinâmica da Fermentação Alcoólica no Processo em Batelada Alimentada", Dissertação de Mestrado, Faculdade de Engenharia Química, 2008. 
[3] A.E. Bryson, Y.C. Ho, "Applied Optimal Control", Hemisphere Publishing, Washington, 1975.

[4] K.E. Brenan, S.L. Campbell, L.R. Petzold, "Numerical Solution of Initial Value Problems in Differential Algebraic Equations", Classics in Applied Mathematics, SIAM Philadelphia, 1996.

[5] E.K. Donald, "Optimal Control Theory: an Introduction", Dover Publications, 2004.

[6] S. Dreyfus, Variational problems with inequality constraints, Journal of Mathematical Analysis and Applications, 4 (1962), 297-308.

[7] W.F. Feehery, "Dynamic Optimization with Path Constraints", Thesis, Massachusetts Institute of Technology, 1998.

[8] H.P. Geering, "Optimal Control with Engineering Applications", Springer Verlag, 2007.

[9] J. Hong, Optimal substrate feeding policy for a fed batch fermentation with substrate and product inhibition kinetics, Biotechnology and Bioengineering, XXVIII (1986), 1421-1431.

[10] F.S. Lobato, "Abordagem Mista para Problemas de Otimização Dinâmica", Dissertação de Mestrado, Faculdade de Engenharia Química, Universidade Federal de Uberlândia, 2004.

[11] F.S. Lobato, L.C. Oliveira-Lopes, V.V. Murata, Optimal feed policy for fedbath fermentation with events identification based on switching structures, XXII IACChE (CIIQ)2006/VCAIQ, (2006).

[12] J.S. Logsdon, L.T. Biegler, Accurate solution of diferential-algebraic optimization problems, Ind. Eng. Chem. Res., 28 (1989), 89-101.

[13] J.M. Modak, H.C. Lim, Y.J. Tayeb, General characteristics of optimal feed rate profiles for various fed-batch fermentation processes, Biotechnology and Bioengineering, XXVIII (1986), 1396-1407.

[14] J.M. Modak, H.C. Lim, Feedback optimization of fed-batch fermentation, Biotechnology and Bioengineering, XXX (1987), 528-540. 